\title{
Growth and Optical Applications of Centimeter-Long ZnO Nanocombs
}

\author{
Ke Yu', Qiuxiang Zhang ${ }^{2}$, Jin Wu', Lijun $\mathrm{Li}^{1}$, Yu'e Xu${ }^{1}$, Shaohua Huang ${ }^{3}$, and Ziqiang Zhu ${ }^{1}(\varangle)$ \\ ${ }^{1}$ Key Laboratory for Polar Materials and Devices of Ministry of Education and Department of Electronic Engineering, East China \\ Normal University, Shanghai 200241, China \\ ${ }^{2}$ Department of Electronic Engineering, Shanghai Jianqiao College, Shanghai 201319, China \\ ${ }^{3}$ Surface Physics Laboratory, Department of Physics, Fudan University, Shanghai 200433, China \\ Received: 8 May 2008/ Revised: 24 July 2008/Accepted: 24 July 2008 \\ CTsinghua Press and Springer-Verlag 2008. This article is published with open access at Springerlink.com
}

\begin{abstract}
Ultralong $\mathrm{ZnO}$ nanocombs have been synthesized on silicon substrates with a high growth rate of $\sim 7 \mu \mathrm{m} / \mathrm{s}$ using a simple "thermal evaporation and condensation" method promoted by $\mathrm{Cu}$ catalysts. The lengths of the $\mathrm{ZnO}$ nanocombs range from several millimeters to more than one centimeter and the diameters of the branches are about $300 \mathrm{~nm}$. The growth mechanism of the ultralong nanocombs and the catalytic behavior of the copper are discussed. The nanocombs were readily separated and their applications as optical polarizer and grating were investigated. The results show that the ultralong $\mathrm{ZnO}$ nanocombs can act as effective optical components in miniaturized integrated optics systems.
\end{abstract}

\section{KEYWORDS}

Zinc oxide nanocombs, thermal evaporation, polarizer, grating

\section{Introduction}

In order to take full advantage of the unique properties of nanostructures in a macroscale environment, one challenging idea is to controllably fabricate functional nanostructures having macroscopic dimensions. Zinc oxide $(\mathrm{ZnO})$, one of the most important functional semiconductor materials with a direct wide band gap of $3.37 \mathrm{eV}$ and a large exciton binding energy of $60 \mathrm{meV}$, has high mechanical strength, thermal stability, and chemical stability [1]. Recently, ZnO comb-like structures, in which nanowires are distributed in an ordered manner on one or both sides of the backbone nanobelt, have attracted considerable attention because their unique geometry is particularly promising for making nanodevices. $\mathrm{ZnO}$ nanocombs have been used to make nanocantilevers [2], UV laser arrays [3], optical gratings [4], and other devices. However, the majority of previous studies have focused on the synthesis and applications of $\mathrm{ZnO}$ nanocombs of microscale length and studies of nanocombs with macroscale lengths have rarely been reported. Therefore, there has been great interest in creating ultralong, continuous nanostructures for applications where their properties coupled with their extended lengths will enable new technological developments. In recent years, ultralong carbon nanotubes (CNTs) and $\mathrm{ZnO}$ nanobelts have been successfully prepared and applied in many fields

Address correspondence to zqzhu@ee.ecnu.edu.cn

\section{算 Springer}


such as mechanics and electronics [5-7]. Here we report the synthesis of centimeter-long $\mathrm{ZnO}$ nanocombs, in large yields, with a high growth rate of $\sim 7 \mu \mathrm{m} / \mathrm{s}$ using a simple one-step "thermal evaporation and condensation" method promoted by $\mathrm{Cu}$ catalysts. The lengths of the $\mathrm{ZnO}$ nanocombs range from several millimeters to more than one centimeter and the diameters of the branches are 200-400 nanometers. The macro-dimensional size of the nanocombs makes the separation of a single nanocomb a relatively easy task. Furthermore, optical experiments demonstrate that the ultralong nanocombs can be used to make effective optical polarizers, optical gratings, and other components.

\section{Experimental}

Nanocomb growth was performed in a conventional horizontal quartz tube furnace at atmospheric pressure. The diameter and length of the tube were $4 \mathrm{~cm}$ and $140 \mathrm{~cm}$, respectively. In the first step, a suitable background for growth of macroscopic $\mathrm{ZnO}$ nanostructures was prepared by heating graphite powder (purity 99.99\%) and $\mathrm{CuO}$ powder (purity $99.9 \%$ ) at $900{ }^{\circ} \mathrm{C}$ for about $3 \mathrm{~h}$ under flowing $\mathrm{Ar}$ $(0.6 \mathrm{~L} / \mathrm{min})$. This process was repeated many times until the downstream of the tube wall became claretred (the color of $\mathrm{Cu}$ ). A reactant mixture of $\mathrm{ZnO}$ powder, graphite powder, and $\mathrm{CuO}$ powder with mass ratio of 2:3:1 was then placed at the end of a long quartz boat. In the same boat, a clean Si substrate was placed $10 \mathrm{~cm}$ downstream from the reactant mixture in order to collect the products. The entire assembly was placed in the center of a preheated tube furnace at $900{ }^{\circ} \mathrm{C}$. After reaction for $40 \mathrm{~min}$ under a constant high purity Ar flow with a rate of $0.6 \mathrm{~L} / \mathrm{min}$, the quartz boat with the Si substrate was taken out from the furnace and cooled down to room temperature. A large quantity of centimeter-long wool-like $\mathrm{ZnO}$ was found deposited on the Si substrate. The same product could also be seen on the walls of the quartz tube.

\section{Results and discussion}

Figure 1 shows typical optical photographs of the
$\mathrm{ZnO}$ products. It can be seen that a large quantity of wool-like $\mathrm{ZnO}$ material had grown quasiperpendicular to the Si substrate, with lengths from several millimeters to more than one centimeter. An individual $1.1 \mathrm{~cm}$ long $\mathrm{ZnO}$ sample, which was separated with the assistance of a needle, is shown in the inset of Fig. 1. The longest observed nanocomb was longer than $2 \mathrm{~cm}$, corresponding to a growth rate of more than $8 \mu \mathrm{m} / \mathrm{s}$. It was found that the longer products became curved while the shorter ones were relatively straight with high structural quality. Figures 2(a)-(d) show scanning electron microscopy (SEM) images of the $\mathrm{ZnO}$ samples. Many $\mathrm{ZnO}$ comblike structures with dense nanowire branches grown on one side of the nanoribbons were observed (Fig. 2(a)). The widths of the nanoribbons are 20-40 $\mu \mathrm{m}$ and the lengths of the nanowire branches are in the range of $30-60 \mu \mathrm{m}$. Figure 2(b) shows part of a single ultralong $\mathrm{ZnO}$ nanocomb placed on the $\mathrm{Si}$ wafer. High magnification SEM images (Figs. 2(c), (d)) reveal that the nanocomb contains several tens of very straight, perfectly aligned and evenly spaced nanowires. The diameters of the nanowires generally range from 300 to $400 \mathrm{~nm}$ and the spacings are 200$500 \mathrm{~nm}$, which corresponds to a periodicity of 500 $900 \mathrm{~nm}$ and a wire density of 1100-2000 wires/ mm.

The composition of a nanocomb on the Si wafer was examined using EDX attached to the SEM. Figure 2(e) shows the $\mathrm{Zn}, \mathrm{O}$, and $\mathrm{Cu}$ elemental mapping of the surface of the nanocomb. It can be clearly seen that the two elements $\mathrm{Zn}$ and $\mathrm{O}$ are

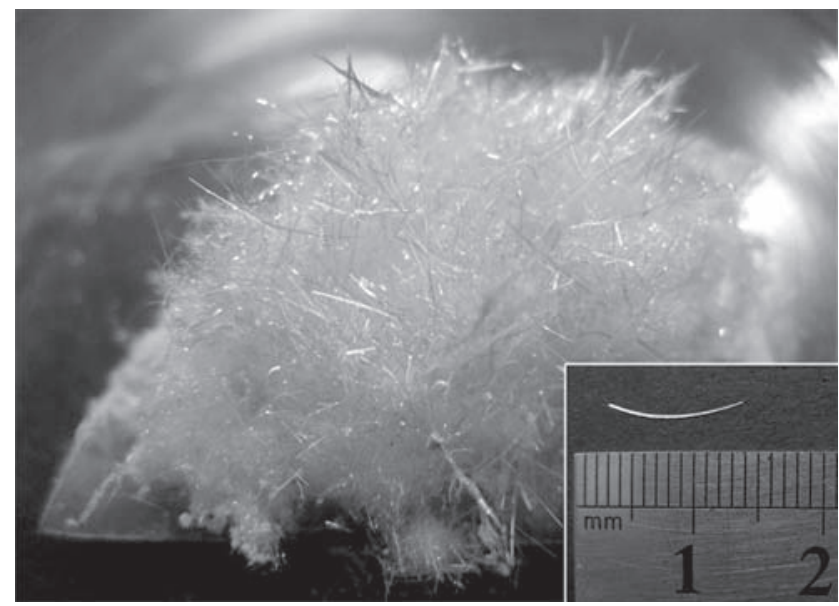

Figure 1 Photographs of the macro-length $\mathrm{ZnO}$ nanocomb structures grown on the Si substrate. The inset is an individual $1.1 \mathrm{~cm}$ long ZnO nanocomb 


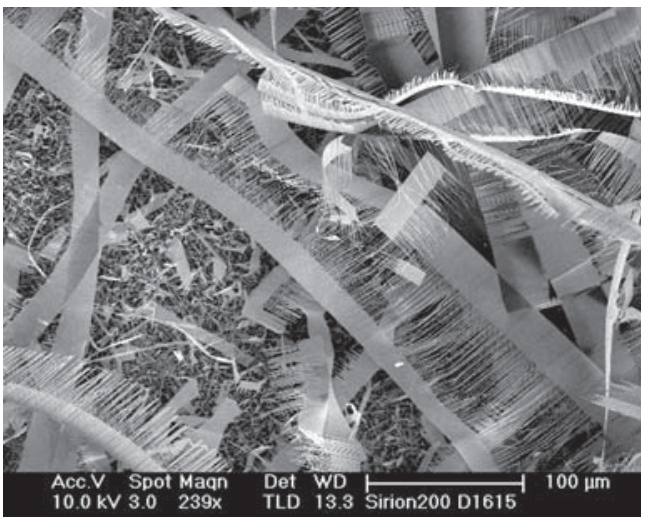

(a)

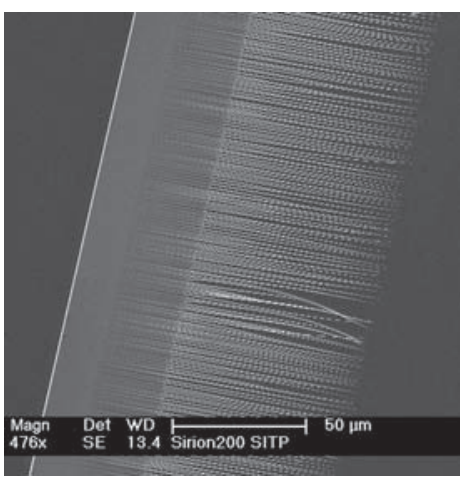

(c)
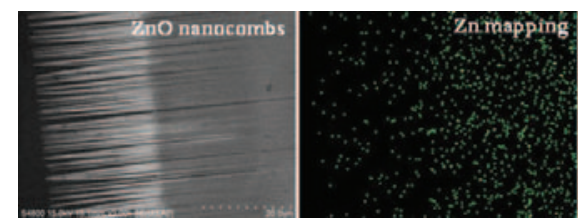

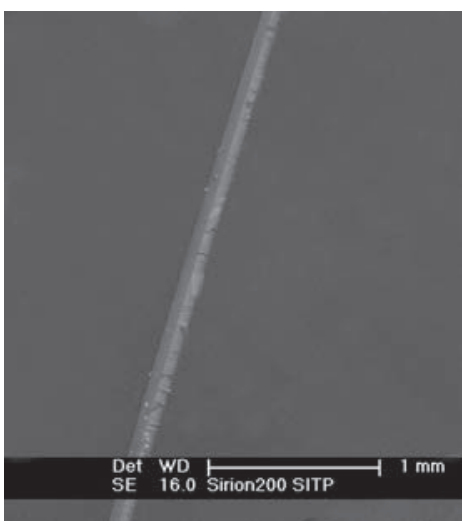

(b)

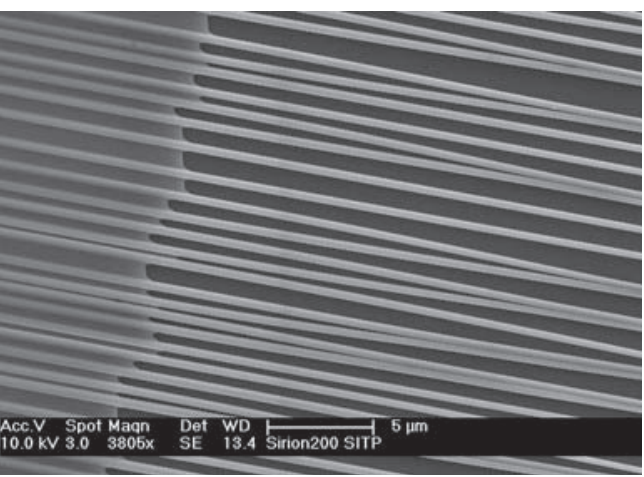

(d)

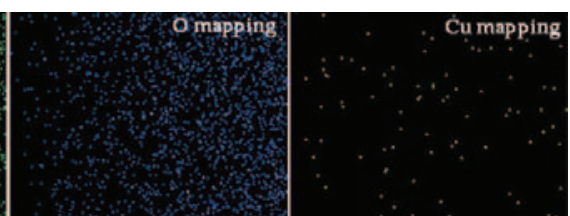

(e)

Figure 2 (a) SEM images of $\mathrm{ZnO}$ nanocombs. (b) Low, (c) medium, and (d) high magnification SEM images of a single $\mathrm{ZnO}$ nanocomb. (e) SEM image of a $\mathrm{ZnO}$ nanocomb and $\mathrm{Zn}, \mathrm{O}$, and Cu elemental mapping of the surface of the nanocomb

uniformly distributed over the whole nanocomb, with the content of $\mathrm{O}$ being a little higher than that of $\mathrm{Zn}$. $\mathrm{Cu}$ is randomly distributed on the surface of the $\mathrm{ZnO}$ nanocombs with a much lower content. The relatively high $\mathrm{O}$ content may be a result of the combined contributions of $\mathrm{O}$ in the surface oxide layer of the Si substrate and that in copper oxide nanoparticles, together with diffusion and adsorption of oxygen on the nanocomb after exposure to air.

Figure 3 shows TEM images of the ultralong $\mathrm{ZnO}$ nanocombs. Figures 3(a) and 3(b) display the initially grown and the perfectly formed $\mathrm{ZnO}$ nanocombs, respectively. The diameter of the branches of the nanocombs is about $350 \mathrm{~nm}$, which is in good agreement with the SEM results. From the TEM images, it can be seen that there are some catalyst particles present on the surface of the nanocombs (marked by arrows). These catalyst particles can be assumed to play an important role in the growth of the nanocombs. Figures 3(c) and (d) respectively show highresolution TEM images taken in areas $\mathbf{a}$ and $\mathbf{b}$ (see Fig. 3(b)) on a branch of the nanocomb. Figure 3(c) clearly shows the (0001) atomic planes (with a separation of 0.52 $\mathrm{nm}$ ) perpendicular to the nanowire branch axis and parallel to the comb ribbon long axis, confirming $\langle 0001\rangle$ and $\langle 01 \overline{1} 0\rangle$ to be the preferred growth direction for the wurtzite $\mathrm{ZnO}$ nanowires and ribbons, respectively, as well as the single crystalline nature of the entire comb structure. Figure 3(d) suggests that $\mathrm{Cu}_{2} \mathrm{O}$ nanoparticles $\sim 7 \mathrm{~nm}$ in size are adsorbed on the surface of the branch of the $\mathrm{ZnO}$ nanocombs since the set of regular lattice spacings of ca. 0.31 $\mathrm{nm}$ corresponds to the separation between two (11̄0) planes of cubic $\mathrm{Cu}_{2} \mathrm{O}$.

The morphology of the products was affected by several experimental parameters including the composition of the reaction mixture, the vapor pressure of $\mathrm{Zn}$, and the growth temperature. By adjusting the experimental parameters, several types of $\mathrm{ZnO}$ nanostructures with different macroscopic appearance, such as ultralong $\mathrm{ZnO}$ nanobelts, fractal $\mathrm{ZnO}$ dendrites, and hierarchical $\mathrm{ZnO}$ hairbrushes were synthesized, as shown in Fig. 4.

A variety of mechanisms for the growth of $\mathrm{ZnO}$ nanocombs have been proposed $[2,8]$. Here, we mainly discuss the reasons why the $\mathrm{ZnO}$ nanocombs can grow to macroscopic size. Comparing the growth conditions in previous experiments that produced shorter nanocombs with those in our experiments, we find that the carrier gas, temperature, and

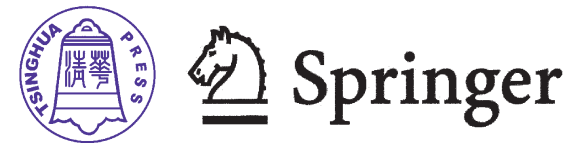


other experimental parameters are similar to those employed elsewhere. The only significant difference is in the reactants. In our experiments, $\mathrm{CuO}$ powder was added to the mixture of $\mathrm{ZnO}$ and graphite powder, whilst most previous workers used $\mathrm{ZnO}$ powder [8], Zn powder [9] or a mixture of $\mathrm{ZnO}$ and

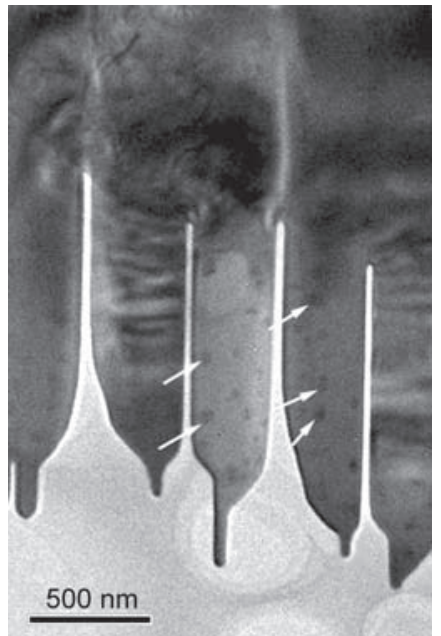

(a)

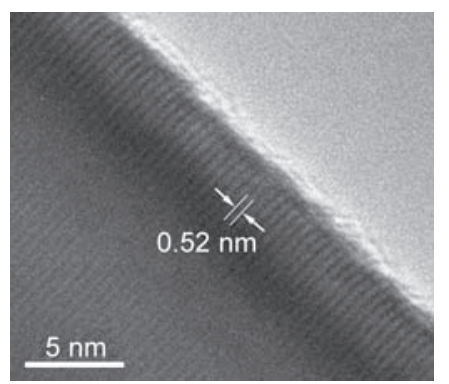

(c)

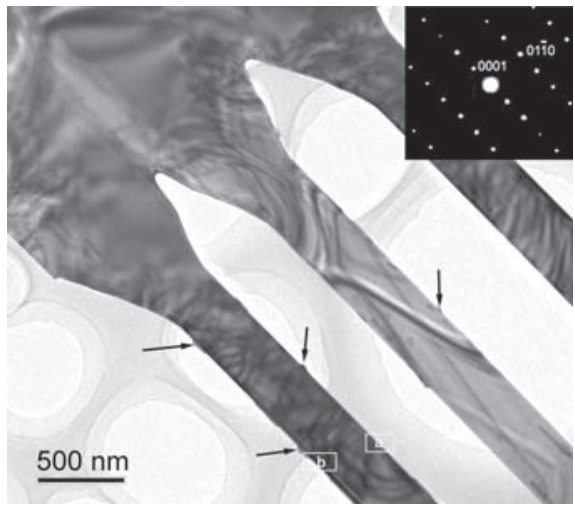

(b)

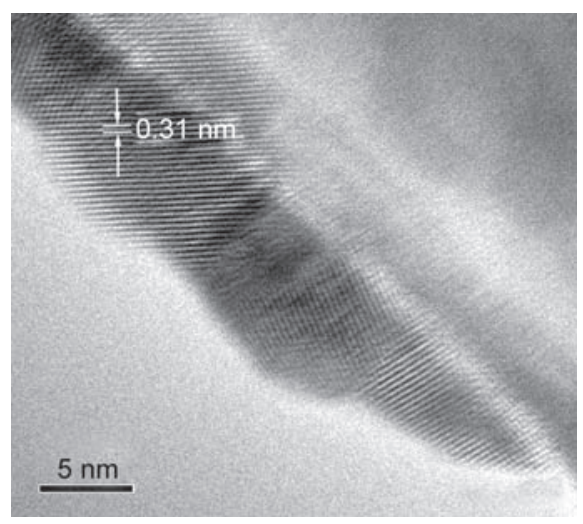

(d)
Figure 3 TEM and high-resolution TEM images of the ZnO nanocombs. (a) The initially grown, and (b) the perfectly formed $\mathrm{ZnO}$ nanocombs, with some catalyst particles present on the surface of the nanocombs (indicated by the arrows). (c) A high-resolution TEM image recorded in area a of the nanocomb in (b). (d) A high-resolution TEM image of $\mathrm{Cu}_{2} \mathrm{O}$ nanoparticles adsorbed on the surface of the nanocomb branch in area $\mathbf{b}$ of the nanocomb in (b) carbon powder [10] as the reactants. This strongly suggests that adding $\mathrm{CuO}$ to the reaction mixture is of critical importance in the formation of the ultralong $\mathrm{ZnO}$ nanostructures observed in our experiments.

At high reaction temperatures, $\mathrm{CuO}$ powder is first reduced to metallic $\mathrm{Cu}$ particles by graphite powder. A similar thermal carbon reduction also occurs with $\mathrm{ZnO}$ powder. The following chemical reactions take place during the thermal evaporation process: $\mathrm{CuO}+\mathrm{C}=\mathrm{Cu}+\mathrm{CO} ; \mathrm{ZnO}+\mathrm{C}=$ $\mathrm{Zn}+\mathrm{CO}$. According to the $\mathrm{Cu}-\mathrm{Zn}$ binary phase diagram, $\mathrm{Zn}$ has a large solubility in $\mathrm{Cu}$ as a solid solution. This ensures that the reduced $\mathrm{Zn}$ atoms can easily dissolve in the $\mathrm{Cu}$ particles to form a $\mathrm{Cu}-\mathrm{Zn}$ eutectic phase which has a higher melting point than the pure zinc phase. Therefore, fewer $\mathrm{Zn}$ atoms are available to evaporate to form a layer of $\mathrm{ZnO}$ nuclei on the clean silicon substrate in the initial stages of deposition. The magnitude and density of $\mathrm{ZnO}$ nuclei on the silicon substrate are therefore significantly reduced compared with the situation without added $\mathrm{CuO}$ powder. As the reaction proceeds, more $\mathrm{Zn}$ atoms are reduced and dissolve into the $\mathrm{Cu}$ particles, and the percentage of $\mathrm{Zn}$ in the $\mathrm{Cu}-\mathrm{Zn}$ binary phase increases resulting in a significant drop in the melting point of the $\mathrm{Cu}-\mathrm{Zn}$ eutectic phase. As a result, the $\mathrm{Cu}-\mathrm{Zn}$ eutectic phase evaporates generating the corresponding

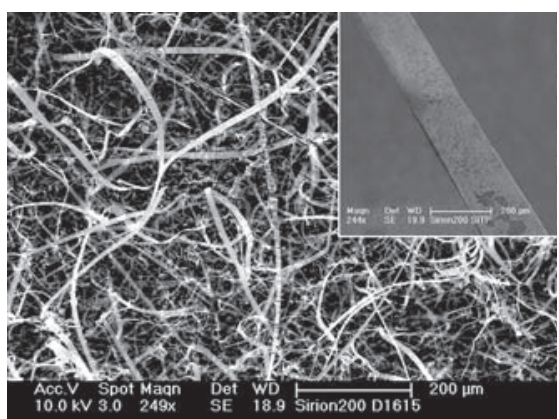

(a)

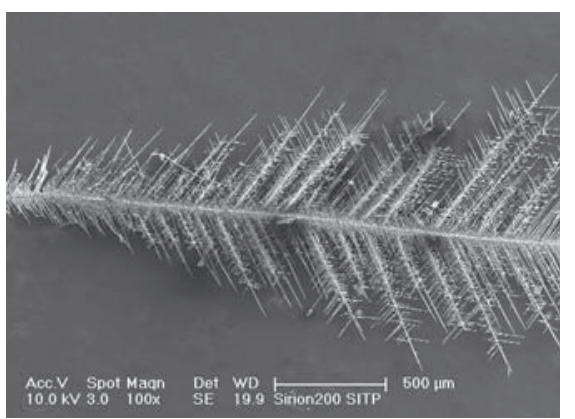

(b)

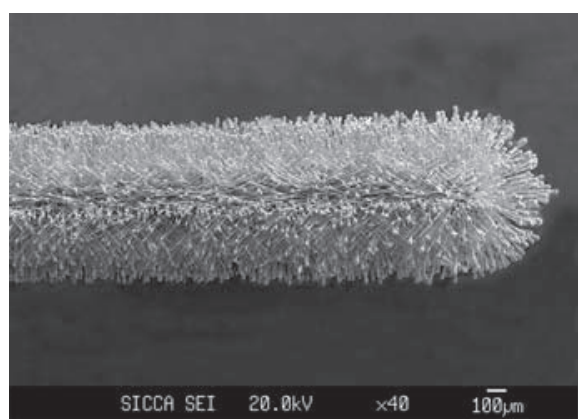

(c)

Figure 4 SEM images of the $\mathrm{ZnO}$ nanostructures with macroscopic appearance: (a) ultralong ZnO nanobelts; (b) fractal ZnO dendrites; (c) hierarchical ZnO hairbrushes 
$\mathrm{Zn}$ vapor with a small quantity of $\mathrm{Cu}$ vapor. During the transition period, the oxygen originating from the residual oxygen in the quartz tube condenses together with the generated $\mathrm{Zn}$ vapor in the lowtemperature region and forms liquid droplets of the $\mathrm{Zn}-\mathrm{Cu}-\mathrm{O}$ eutectic phase. After supersaturation of elemental $\mathrm{Zn}$ and $\mathrm{O}, \mathrm{ZnO}$ nanoarchitectures begin to form on the $\mathrm{ZnO}$ nuclei, and then gradually develop into ultralong $\mathrm{ZnO}$ nanostructures. The residual $\mathrm{Cu}$ adheres to the surface of the ultralong $\mathrm{ZnO}$ nanostructures in the form of nanoparticles. $\mathrm{Cu}$ can be considered as a catalyst for the growth of the ultralong $\mathrm{ZnO}$ nanostructures. Similar studies using $\mathrm{Cu}$ as a catalyst in the preparation of semiconductor nanostructures and ultralong single-walled carbon nanotubes have been reported [11-13]. Figure 5 shows a schematic illustration of the growth processes of the $\mathrm{ZnO}$ nanostructures in the presence and absence of $\mathrm{CuO}$ in the reactant mixture.

On the basis of the growth process outlined above, there are three factors that determine the growth mechanism of the ultralong $\mathrm{ZnO}$ nanostructures. First of all, the formation of the $\mathrm{Cu}-\mathrm{Zn}$ eutectic phase controls the release of $\mathrm{Zn}$ and significantly decreases the magnitude and density of $\mathrm{ZnO}$ nuclei on the silicon substrate in the very early stages. Secondly, only when the concentration of $\mathrm{Zn}$ reaches a certain percentage, can $\mathrm{Zn}$ vapor and a small amount of $\mathrm{Cu}$ vapor be generated. Adding $\mathrm{CuO}$ powder to the reactant mixture has a marked effect on the release of $\mathrm{Zn}$ atoms and for this reason, $\mathrm{Zn}$ vapor can be evenly transported to the substrate in a stable manner. Lastly, with the formation of liquid droplets of the $\mathrm{Zn}-\mathrm{Cu}-\mathrm{O}$ eutectic phase, it becomes much easier to absorb and accumulate $\mathrm{Zn}$ vapor. The HRTEM and EDX analyses of the samples indicate that some elemental $\mathrm{Cu}$ exists on the surface of the $\mathrm{ZnO}$ ultralong nanostructures, which supports our proposal of the existence of the $\mathrm{Cu}-\mathrm{Zn}$ eutectic phase and the liquid droplets of the $\mathrm{Zn}-\mathrm{Cu}-\mathrm{O}$ eutectic phase during the growth process.

The $\mathrm{Cu}(110)$ and $\mathrm{Cu}(001)$ surfaces show a strong tendency to reconstruct under the chemisorption of oxygen, and a "missing row" model, has been proposed and confirmed by different techniques [14-16]. Thus, it can be proposed that the $\{110\}$ and $\{001\}$ surfaces of the $\mathrm{Cu}$ nanoparticle catalyst have undergone a missing-row-type reconstruction induced by oxygen chemisorption, and this finally

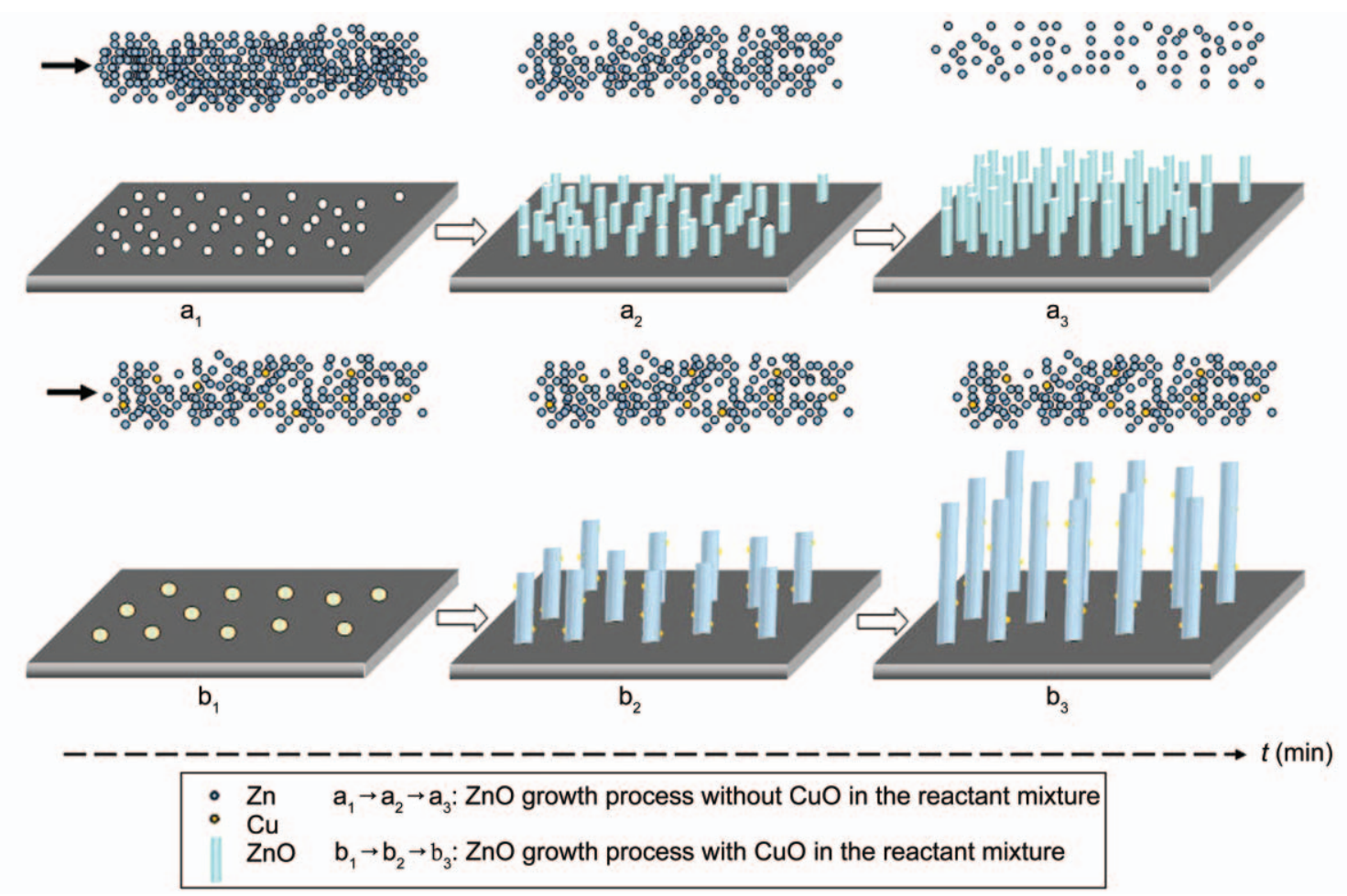

Figure 5 Schematic illustration of the growth process of the $\mathrm{ZnO}$ nanostructures with and without $\mathrm{CuO}$ in the reactant mixture

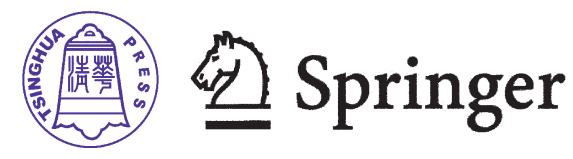


leads to the formation of $\mathrm{Cu}_{2} \mathrm{O}$ nanoparticles on the surface of the $\mathrm{ZnO}$ nanocombs, in agreement with the HRTEM observations.

In order to confirm that it is indeed $\mathrm{Cu}$ that acts as the catalyst, we undertook many careful investigations. Firstly, we used new quartz tubes for the $\mathrm{Cu}$-catalyzed experiments in order to avoid contamination. Secondly, without adding $\mathrm{CuO}$ powder to the reaction mixture, we repeatedly did a series of experiments involving adjusting the experimental parameters, and did not find any trace of the formation of ultralong $\mathrm{ZnO}$ nanostructures. Finally, we carried out parallel experiments using a small amount of $\mathrm{Cu}(99.999 \%)$ powder instead of $\mathrm{CuO}$ powder and found that $\mathrm{ZnO}$ nanostructures with a macroscopic appearance were still formed. From all the above results, we can be confident that it is indeed the $\mathrm{Cu}$ that catalyzes the growth of the ultralong $\mathrm{ZnO}$ nanocombs.

The nanocombs can be regarded as potential optical components in miniaturized integrated optics systems, such as polarizers and diffraction gratings. As an example, we constructed a single $\mathrm{ZnO}$ nanocomb UV polarizer. The intensity of the 325 $\mathrm{nm}$ line of a He-Cd laser was recorded by a Si charge coupled detector as shown in Fig. 6. The polarized laser light $\left(\mathrm{P}_{\text {laser }}\right)$ was focused on the surface of an $\mathrm{Si}$ wafer and reflected back, passing through the $\mathrm{ZnO}$

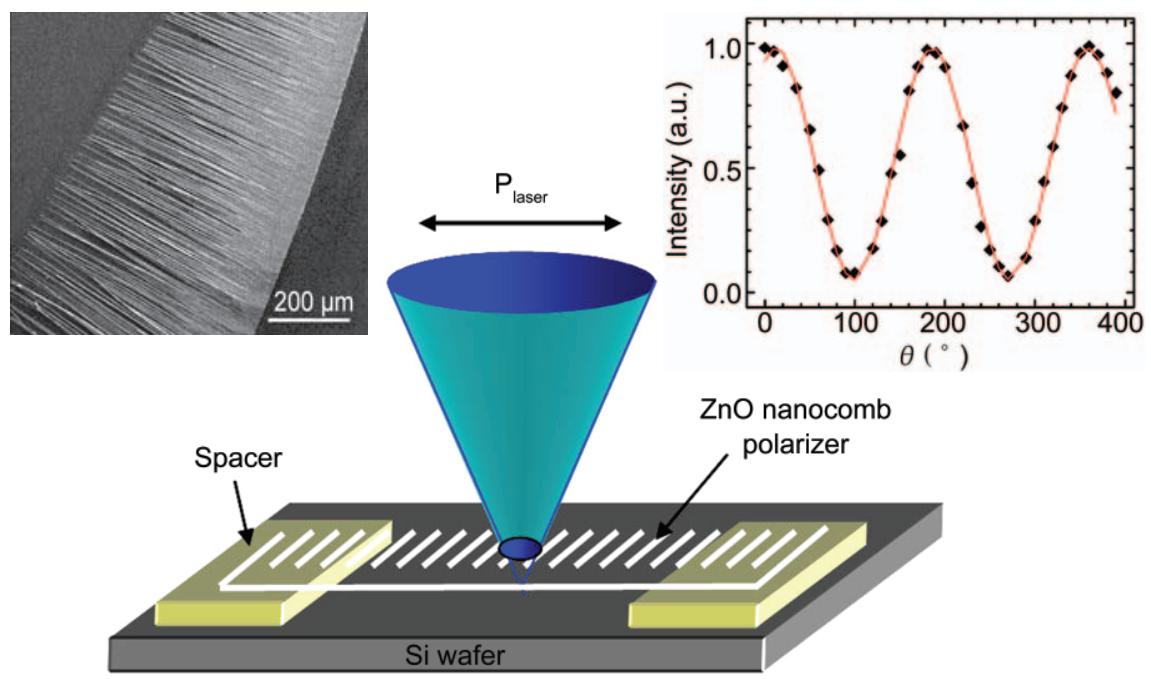

Figure 6 Schematic diagram of the experimental setup for polarization measurements. The upper left inset is a $\mathrm{ZnO}$ nanocomb polarizer. The upper right inset is the normalized intensity plotted against the angle $\theta$ between the polarization direction of the laser light and the axis of the backbone of the $\mathrm{ZnO}$ nanocomb polarizer nanocomb polarizer twice before being collected by a CCD detector. When a beam of light passes through the polarizer, photons with polarization direction parallel to the axis of the branches of the nanocomb are absorbed, whereas those with perpendicular polarization pass through it. The upper right inset of Fig. 6 shows the normalized intensity plotted against the angle between the polarization direction of the laser light at $325 \mathrm{~nm}$ and the $\mathrm{ZnO}$ nanocomb polarizer, which is in good agreement with Malus's law $\left(I=I_{0} \cos ^{2} \theta\right.$, dotted line).

A schematic diagram of the $\mathrm{ZnO}$ nanocomb diffraction measurement system is shown in Fig. 7 (a). An Olympus microscope was used to focus the incident laser light to a spot approximately $5-8 \mu \mathrm{m}$ in diameter, and lasers of different wavelength (a HeNe laser at $632.8 \mathrm{~nm}$ (red light) and a GaN laser at 407 $\mathrm{nm}$ (blue light)) were employed to acquire diffraction images with different resolution ability. The laser entered the microscope through the back port and was directed onto the $\mathrm{ZnO}$ combs, which were supported on a glass cover slip, by a $50 \times$ objective lens. The use of the microscope permitted accurate alignment of the laser spot on the nanowire array of the comb. A screen was positioned at a known distance below the sample stage in order to observe the diffracted beam and to allow for measurement of the separation between the central and diffracted spots. Figures 7(c) and (d) show the diffraction patterns from a single $\mathrm{ZnO}$ nanocomb with a period of $\sim 800 \mathrm{~nm}(\sim 300 \mathrm{~nm}$ in width, with a spacing of $\sim 500$ $\mathrm{nm})$, as estimated from the SEM image in Fig. 7(b), using the lasers of different wavelength. It can be seen that the incident laser beam was diffracted into one zero-order central beam and two first-order side beams with approximately equal intensity, showing a strong diffraction effect.

Since the incident light is perpendicular to the comb surface, the diffraction behavior of the nanocomb grating can be 


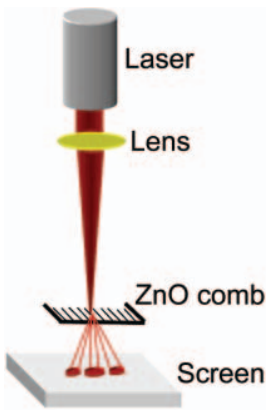

(a)

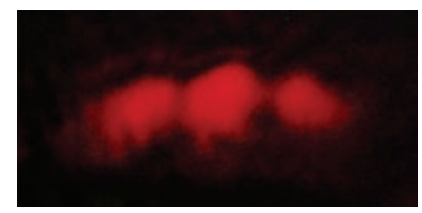

(c)

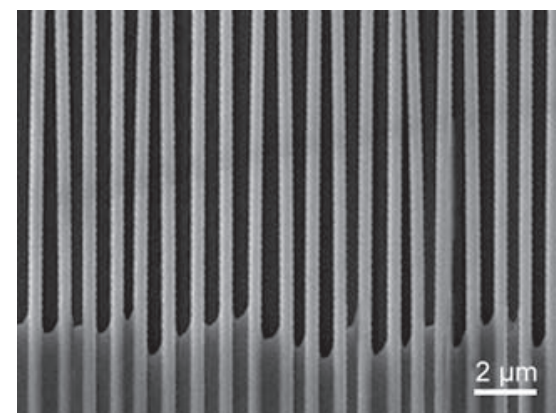

(b)

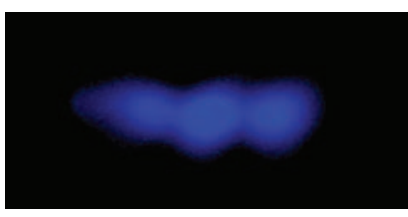

(d)

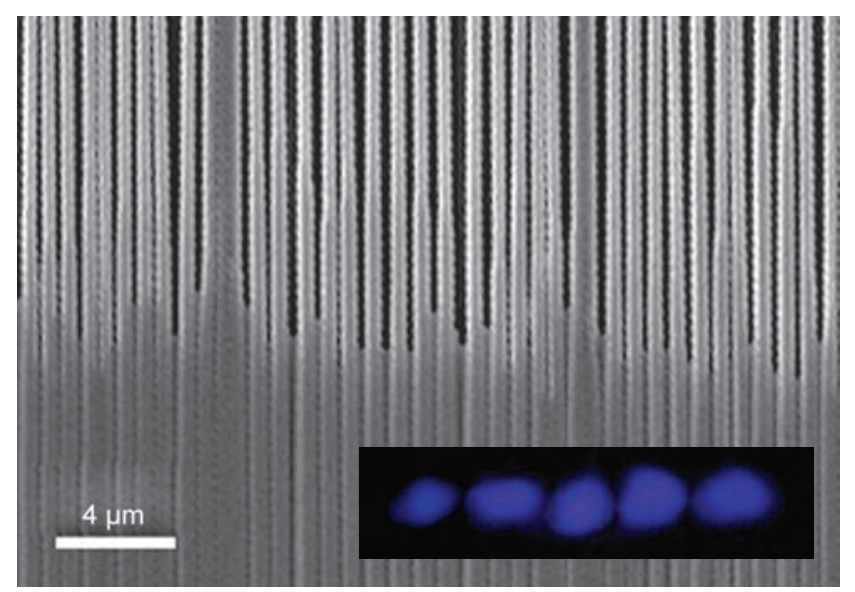

(e)

Figure 7 (a) Schematic diagram of the experimental setup for diffraction measurements. (b) SEM image of a ZnO nanocomb grating with a period of $\sim 800 \mathrm{~nm}(\sim 300 \mathrm{~nm}$ in width with a spacing of $\sim 500$ $\mathrm{nm}$ ). Diffraction patterns generated by the $\mathrm{ZnO}$ comb grating under the irradiation of (c) a red laser beam and (d) a blue laser beam, respectively. (e) SEM image of a $\mathrm{ZnO}$ nanocomb grating with a period of $\sim 850 \mathrm{~nm}(\sim 600 \mathrm{~nm}$ in width with a spacing of $\sim 250 \mathrm{~nm})$; the inset is the corresponding diffraction pattern

determined by the simplified grating equation $m \lambda=$ $d \sin \phi$, where $m, d, \lambda$, and $\phi$ are the diffraction order, period of the nanocomb, wavelength of the incident light and diffraction angle with respect to the grating normal, respectively. The calculated diffraction angle $\phi_{1}$ of the first-order diffraction based on the distance between the screen and comb and the separation between the central and diffracted spots, is $\sim 51^{\circ}$ (red light as the light source), or $30^{\circ}$ (blue light as the light source). Substituting $\phi_{1}$ into the grating equation, we obtain $d=815 \mathrm{~nm}$ (for $\phi_{1}=51^{\circ}$ ) or
$814 \mathrm{~nm}$ (for $\phi_{1}=30^{\circ}$ ), respectively. Considering errors in distance measurements, these two values are very close to the predicted period of $800 \mathrm{~nm}$ of the nanocomb from the SEM image.

We also studied the diffraction effect of the comb gratings with the smaller spacing of the nanowire branches. The inset in Fig. 7(e) shows the diffraction patterns generated from a comb with a period of $\sim 850 \mathrm{~nm}(\sim 600 \mathrm{~nm}$ in width with a spacing of $\sim 250$ $\mathrm{nm})$ using a blue laser as the light source. Clearly, the incident laser beam is diffracted into one zero-order central beam, two first-order side beams, and two second-order side beams. The observed separation between the central and diffracted spots also indicates that the $\mathrm{ZnO}$ nanocomb is a promising potential multibeam divider in miniaturized integrated optics systems.

\section{Conclusions}

In summary, we present a simple method to produce centimeter-long nanocombs on a Si substrate. The capability to grow ultralong nanocombs represents a major step forward in the synthesis of macroscopic sized nanostructures. The successful fabrication of a $\mathrm{ZnO}$ nanocomb polarizer and grating suggests promising potential applications of the material in macroscopic photoelectric functional devices.

\section{Acknowledgments}

The authors acknowledge the financial support from the Chinese National Key Basic Research Special Fund (Grant Nos. 2006CB921704 and 2007CB924902), the Specialized Research Fund for the Doctoral Program of Higher Education (Grant No. 20070269016), Chenguang Project of Shanghai Education Development Foundation (Grant No. 2008CGB23), and the Excellent Young Teachers Program of Shanghai Higher Learning Institutions (Grant No. AAYQ0723).

\section{References}

[1] Wang, Z. L.; Kong, X. Y.; Ding, Y.; Gao, P.; Hughes, W. L.; Yang, R.; Zhang, Y. Semiconducting and piezoelectric 
oxide nanostructures induced by polar surfaces. Adv. Funct. Mater. 2004, 14, 943-956.

[2] Wang, Z. L.; Kong, X. Y.; Zuo, J. M. Induced growth of asymmetric nanocantilever arrays on polar surfaces. Phys. Rev. Lett. 2003, 91, 185502.

[3] Yan, H.; He, R.; Johnson, J.; Law, M.; Saykally, R. J.; Yang, P. Dendritic nanowire ultraviolet laser array. J. Am. Chem. Soc. 2003, 125, 4728-4729.

[4] Pan, Z. W.; Mahurin, S. M.; Dai, S.; Lowndes, D. H. Nanowire array gratings with $\mathrm{ZnO}$ combs. Nano Lett. 2005, 5, 723-727.

[5] Zhu, H. W.; Xu, C. L.; Wu, D. H.; Wei, B. Q.; Vajtai, R. P.; Ajayan, M. Direct synthesis of long single-walled carbon nanotube strands. Science 2002, 296, 884-886.

[6] Huang, S.; Maynor, B.; Cai, X. Y.; Liu, J. Ultralong, wellaligned single-walled carbon nanotube architectures on surfaces. Adv. Mater. 2003, 15, 1651-1655.

[7] Wang, W. Z.; Zeng, B. Q.; Yang, J.; Poudel, B.; Huang, J. Y.; Naughton, M. J.; Ren, Z. F. Aligned ultralong ZnO nanobelts and their enhanced field emission. Adv. Mater. 2006, 18, 3275-3278.

[8] Lao, C. S.; Gao, P. X.; Yang, R. S.; Zhang, Y.; Dai, Y.; Wang, Z. L. Formation of double-side teethed nanocombs of $\mathrm{ZnO}$ and self-catalysis of $\mathrm{Zn}$-terminated polar surface. Chem. Phys. Lett. 2005, 417, 359-363.

[9] Shen, G. Z.; Bando, Y.; Chen, D.; Liu, B. D.; Zhi, C. Y.; Golberg, D. Morphology-controlled synthesis of $\mathrm{ZnO}$ nanostructures by a simple round-to-round metal vapor deposition route. J. Phys. Chem. B 2006, 110, 39733978.

[10] Lim, Y. S.; Park, J. W.; Hong, S. T.; Kim, J. Carbothermal synthesis of $\mathrm{ZnO}$ nanocomb structure. Mater. Sci. Eng. $B$ 2006, 129, 100-103.

[11] Yang, W. Y.; Xie, Z. P.; Miao, H. Z.; Zhang, L. G.; An, L. N. Coalescence of nanobranches: A new growth mechanism for single crystal nanobelts. J. Phys. Chem. $B$ 2006, 110, 3969-3972.

[12] Li, S. Y.; Lee, C. Y.; Tseng, T. Y. Copper-catalyzed ZnO nanowires on silicon (100) grown by vapor-liquid-solid process. J. Cryst. Growth 2003, 247, 357-362.

[13] Zhou, W. W.; Han, Z. Y.; Wang, J. Y.; Zhang, Y.; Jin, Z.; Sun, X.; Zhang, Y. W.; Yan, C. H.; Li, Y. Copper catalyzing growth of single-walled carbon nanotubes on substrates. Nano Lett. 2006, 6, 2987-2990.

[14] Onuferko J. H.; Woodruff, D. P. LEED structural study of the adsorption of oxygen on $\mathrm{Cu}\{100\}$ surfaces. Surf. Sci. 1980, 95, 555-570.

[15] Richter, H.; Gerhardt, U. Adsorption sites of oxygen on $\mathrm{Cu}(001)$ and $\mathrm{Ni}(001)$ determined from the shape of the low-energy electron-diffraction spots. Phys. Rev. Lett. 1983, 51, 1570-1572.

[16] Parkin, S. R.; Zeng, H. C.; Zhou, M. Y.; Mitchell, K. A. R. Low-energy electron-diffraction crystallographic determination for the $\mathrm{Cu}(110) 2 \times 1-\mathrm{O}$ surface structure. Phys. Rev. B 1990, 41, 5432-5435. 